\title{
ON FORECASTING THE SUNSPOT NUMBERS
}

J. Kurths ${ }^{1}$ and A. A. Ruzmaikin ${ }^{2}$

1 Zentralinstitut fur Astrophysik, 1501 Tremsdorf G.D.R.

2 IZMIRAN, 142092 Troitsk, Moscow Region, U.S.S.R.

(Received 27 December, 1989)

\begin{abstract}
We have applied a technique recently proposed basing on learning nonlinear dynamies locally to describe the annual sunspot relative numbers. It is proved that the number of past points for prediction should be greater than 4 but less than 10 . This rather siraple approach yields in average relatively good results for shortterm forecasts $(<11 \mathrm{yr})$. Particularly, it predicts that the current cycle no. 22 will reach a very high maximum. However, this approach must be modified in the vicinity of a grand minimum.
\end{abstract}

\section{MOTIVATION}

The problem of forecasting the solar activity is a classical one (ef. Witinskij et al., 1986). Since dynamo models of this process have not been developed on the quantitative level, one has to make predictions directly from the data. Yule (1927) was the first to predict the sunspot numbers using a stochastic autoregressive model. Further attempts viewed the Wolf numbers as a realization of a random process superimposing trends and "seasonal" components (Sneyers and Cugnon, 1986 or Wilson, 1988).

In the last decade a lot of examples in nonlinear dynamies has indicated that irregular behaviour is a manifestation of low-dimensional chaos (Ruelle, 1989). Due to the exponential instability of initially nearby trajectories chaos places a fundamental limit on long-term prognoses, but short-term forecasts might be quite accurate because such systems are deterministic.

Applying an attractor analysis to the Wolf numbers, some evidence to low-dimensional nonlinear dynamics have been yielded (Kurths, 1987, Ajmanova and Makarenko, 1988, Morfill and Voges, 1989). Basing on these results we deal the problem of forecasting the solar activity in general 
and we make predictions of the Wolf numbers for the present solar cycle no. 22.

\section{THE METHOD}

\subsection{Phase Space Reconstruction}

The state of the dynanical system underlying the solar activity is determined by several variables. Their number corresponds to the attractor dimension which has been estimated less than 3 from an analysis of the Wolf numbers W(t). It is possible to reconstruct the dynamical variables from this single time series by using Takens' (1981) proposal of delay-coordinate to form a vector

$\underline{w}(t)=\langle W(t), W(t+q), \ldots, W(t+(m-1) q\rangle\}$

where $m$ is the integer number determining the space in which the attractor if embedded.

\subsection{Forecasting Model}

As was shown by Farmer and Sidorovich (1987) a relationship between a current state and its future

$\underline{w}(t+\Delta t)=f(\underline{w}(t))$

over a short prediction time $\Delta t$ can be approximated by a linear law

$\underline{\underline{u}}(t+\Delta t)=\underline{a} \underline{\underline{w}}(t)+\underline{b}$.

The constant matrix $\underline{a}$ and the vector $\underline{b}$ can be calculated applying the method of least squares. It is clear that $k>m+1$ points at $t$ ' < $t$, which are the nearest spatial neighbours of (t), are needed for this approximation.

\section{FORECASTING THE WOLF NUMBERS}

In this paper we take the annual sunspot relative numbers from 1749 to 1888. The reversals of the magnetic polarity between subsequent oycles are taken into acoount by giving alternating signs to successive cycles (Fig. 1), as proposed by Bracewell (1986).

From the attractor analyses (Kurths, 1987, Ajmanova and Makarenko, 1988, Morfill and Voges, 1989) we have yielded that an embedding dimension $\mathrm{m}=3-4$ and a delay time $r=2-5$ yr are suitable parameters to embed W(t) via (1). Next, we have to choose $k>m+1$ (in fact $k=5-8$ is 


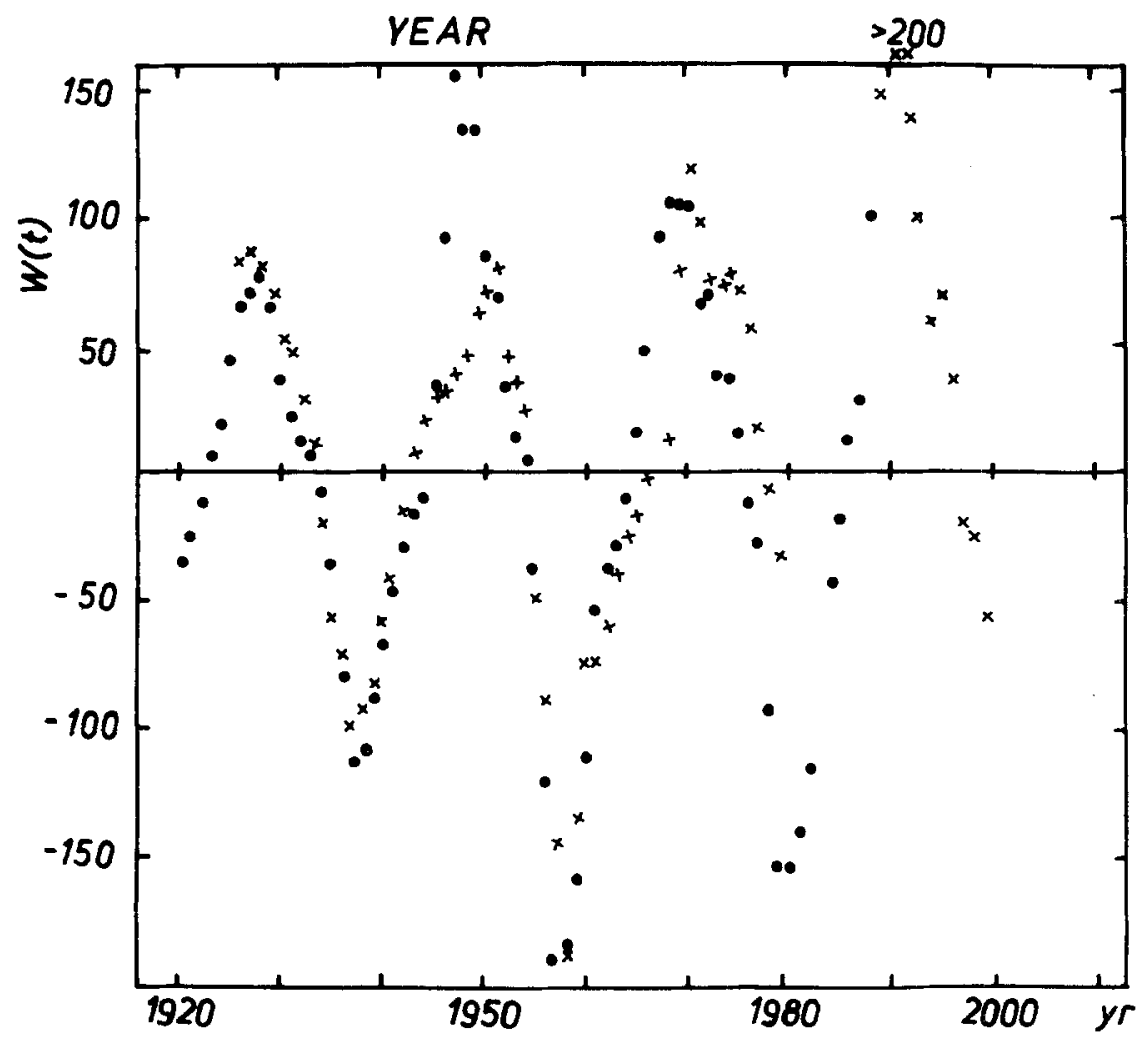

Fig. 1. A part of the annual relative sunspot numbers (dots) and some of their forecasts (crosses) basing on eq. 2

sufficient) spatial neighbours of various reference points and estimate the model parameters $a$ and $b$ for each refecence point as well as for different prediction times $\Delta t$.

The first 175 points $(1749$ - 1923) are used as a "learning" sample to fit the matrices a resp. the vectors $\underline{b}$ in $E q .2$, $i$. e. we "learn" the dynamics inherent the Wolf numbers from this part.

\section{RESULTS}

This method provides relatively Eood predictions (Fig. 1) which reproduce low as well as high maxima in the case of rather short-term forecasts $\left(\Delta t<19 \mathrm{yr}^{\circ}\right)$. Note that this 
procedure assumes homogeneity of the solar attractor. Indeed, this attractor appears to be inhomogeneous meaning, loosely speaking, that a trajectory visits some parts of the attractor more frequently than others. The influence of this fact to the forecast will be studied in a forthcoming paper with Makarenko and Ajmanova.

Applying this technique to a short-term prognosis, we yield indications that the present cycle no. 22 will produce a very high maximum. These results give further support to a chaotic behaviour of the solar activity which precludes long-term predictions. This is the reason why for longer predictions considerable errors in both amplitude and phase occur.

The pronounced manifestations of chaotic behaviour are grand minima like the Maunder minimurn occured over $7 \emptyset$ yr in the 17th century (Eddy, 1983). In order to forecast the solar activity in the vicinity of a grand minimum one should take into account lone-term characteristics of the dynamics, too.

\section{ACKNOWLEDGEMENTS}

We are very much indepted to T. S. Tavastsherna for preparing the data and to N. G. Makarenko and G. K. Ajmanova for valuable discussions.

\section{REFERENCES}

Ajmanova, G. K. and Makarenko, N. G. : 1988, AZ No 1527, 27.

Bracewel1, R. N.: 1986, Nature 323, 516.

Eddy, J. A.: 1983, Solan Phys.

Farrer, J. D. and Sidorovich, J. J.: 1987, Phys. Rey. Lett. 59,845 .

Kurths, J. 1987, Preprint ZIAP 87-Ø2.

Morfill, G. and Voges, W.: 198, Sun in Time, Arizona University Press.

Ruelle, D.: 1989, Chaotic Evolution and Strange Attractors, Notes Lezioni Lincee, Cambridge University Press.s.

Sneyers, R. and CuEnon, P.: 1986, Ann. Geophysicae 4, 81. Takens, F.: 1981, Dynamical Systems and Turbulence, SprinBer Verlag, Berlin.

Wilson, R. M.: 1988, Nature 335, 773.

Witinskij, J. I., Kopecky, M. and Kuklin, G. W. : 1986, Statistika Pjatnoobrazowatelnoj Dejatelnosti Solnsá, NAUKA, Moskau.

Yule, G. U.: 1927, Philos. Trans. Roy. Soc. London. Ser. A. $\underline{\underline{26}}, 267$. 